\title{
ESTUDIO DE PREVALENCIA DE RETINOPATÍA DIABÉTICA EN PACIENTES DIABÉTICOS MEDIANTE RETINÓGRAFO NO MIDRIÁTICO
}

\section{USE OF NON-MYDRIATIC RETINOGRAPHY TO DETERMINE THE PREVALENCE OF DIABETIC RETINOPATHY IN DIABETIC PATIENTS}

\author{
VLEMING EN ${ }^{1}$, CASTRO M${ }^{1}$, LÓPEZ-MOLINA MI ${ }^{1}$, TEUS MA ${ }^{2}$
}

\begin{abstract}
RESUMEN
Objetivo: Conocer la prevalencia y gravedad de la retinopatía diabética $(\mathrm{RD})$ en una muestra poblacional de pacientes diabéticos del área sanitaria 3 mediante el empleo de cámaras no midriáticas (CNM) y telemedicina.

Métodos: Se incluyeron todos los pacientes que acudieron a revisión de fondo de ojo en el área sanitaria 3 de la comunidad de Madrid por presentar diabetes mellitus tipo 1 (DM1), diabetes mellitus tipo no insulinodependientes (DM2NID) y diabetes tipo 2 en tratamiento con insulina (DM2ID). Se realizaron retinografías en tres campos de los dos ojos, que fueron valoradas por un oftalmólogo en el hospital. Se valoró la presencia de retinopatía diabética y su gravedad.

Resultados: En el 79\% de los casos la exploración no mostró signos de RD, en el 9\% RD no proliferativa leve, en el 9\% RD no proliferante moderada, en el $1 \%$ RD no proliferante severa. Esto arroja una prevalencia aproximada del $21 \%$ de RD en la población diabética del área 3. El 12\% de las retinografías fueron no valorables, necesitando exploración por un oftalmólogo.
\end{abstract}

\begin{abstract}
Objectives: To establish the prevalence and severity of diabetic retinopathy (DR) in a sample of patients from «Health Area $3 »$ in the Community of Madrid using non-mydriatic retinography and telemedicine.

Methods: All patients who had their ocular fundus examined due to one of the following conditions were included in the sample: type 1 diabetes (DM1), type 2 non- insulin-dependant diabetes (DM2NID), and type 2 insulin-dependant diabetes (DM2ID). In all cases, 3-field retinographies were taken of both eyes to be evaluated for the presence of DR, and its severity, by an ophthalmologist at the hospital.

Results: No DR was observed in $79 \%$ of the patients examined, $9 \%$ had a mild non-proliferative DR, $9 \%$ had a moderate non-proliferative DR, and $1 \%$ had a severe non-proliferative DR. The estimated prevalence of DR in «Health Area 3» of the Community of Madrid was thus $21 \%$. In $12 \%$ of the examinations the retinographies were considered «low quality», thus requiring a second evaluation at hospital.
\end{abstract}

\footnotetext{
Recibido: 30/5/07. Aceptado: 17/4/09.

Servicio de Oftalmología. Hospital Universitario Príncipe de Asturias. Alcalá de Henares. Madrid. España.

${ }^{1}$ Licenciado en Medicina.

2 Doctor en Medicina. Universidad de Alcalá.

Correspondencia:

Eduardo N. Vleming

C/. Deyanira 17 , portal $1,2 .^{\circ} \mathrm{B}$

28022 Madrid

España

E-mail: e_vleming@yahoo.es
} 
Conclusiones: El uso de cámaras no midriáticas y telemedicina es un método adecuado para el cribado de la RD que en la población afecta de diabetes mellitus.

Palabras clave: Retinopatía diabética, Diabetes mellitus, cámara no midriática, estudio poblacional, neovascularización.
Conclusions: The use of non-mydriatic retinography and telemedicine is an adequate method for the screening of DR among the diabetic population (Arch Soc Esp Oftalmol 2009; 84: 231-236).

Key words: Diabetic retinopathy, diabetes mellitus, non-mydriatic camera, population study, neovascularization.

\section{INTRODUCCIÓN}

La diabetes es la causa más frecuente de ceguera legal en los países desarrollados entre los 20 y los 64 años (1). La detección precoz de los pacientes con retinopatía diabética (RD) susceptible de tratamiento, permitiría disminuir la incidencia de complicaciones oculares graves y pérdida visual severa, así como un ahorro en términos económicos (2). Por eso se recomiendan a los pacientes diabéticos revisiones oftalmológicas periódicas. La prevalencia de la diabetes Mellitus (DM) en nuestro medio hace que la visita rutinaria de estos pacientes suponga un alto coste en recursos. Si el cribado de la patología diabética se realizase fuera de la consulta oftalmológica, se podría optimizar el uso de la misma para la patología más grave. El «gold Standard» según el «Early Treatment Diabetic rethinopathy Study» (ETDRS) para el cribado de la RD es el uso de de 7 fotografías de FO que demostró mayor sensibilidad y especificidad que el examen clínico con oftalmoscopia indirecta $(3,4)$.

En los últimos años se ha venido estudiando la utilidad de las cámaras no midriáticas (CNM) con este objetivo, demostrando la misma eficacia que el ETDRS (5-12). El EURODIAB IDDM recomienda el uso de tres retinografías planas de $45^{\circ}$ (13).

No existe en el momento actual ningún estudio que se centre en la prevalencia de RD en un área sanitaria española completa, no conociéndose por tanto la prevalencia real de dicha enfermedad en nuestro medio. Nuestro trabajo es un estudio piloto que pretende definir la prevalencia de RD en la población diabética controlada por el Servicio de Salud Madrileño en el área sanitaria 3 de la comunidad de Madrid. Según datos del censo de la comunidad de Madrid la población del área 3 en 2008 era de 379.000 habitantes, de modo que se puede estimar el número de diabéticos en alrededor de 19.000 .

\section{SUJETOS, MATERIAL Y MÉTODOS}

Este es un estudio prospectivo, observacional, en el que se han incluido todos los pacientes remitidos por su médico de atención primaria y por el endocrinólogo para revisión de fondo de ojo, de manera consecutiva, por padecer diabetes mellitus entre el día 1 de octubre de 2006 y 30 de marzo de 2007, un total de 1.393 pacientes. Los pacientes acudieron a consulta en los dos Centros de Especialidades Periféricos (CEP) del Área III. Allí fueron realizadas tres retinografías de 45 grados de cada fondo de ojo, siguiendo el protocolo de la Joslin Vision Network (12), es decir, una centrada en polo posterior, y otras dos más, cada una de ellas centrada en media periferia superior y nasal repectivamente. Además, y por motivo de seguridad y de identificación del paciente, realizamos una cuarta fotografía del iris de cada ojo. Las retinografías y fotografías se realizaron con retinógrafos no midriáticos tipo TRCNW6S de la casa TOPCON, conectados a la intranet del Hospital Universitario Príncipe de Asturias (HUPA).

Una vez realizadas las retinografías por un optometrista, un oftalmólogo de la unidad de retina y vítreo del HUPA evaluó las imágenes tomadas de cada fondo de ojo, para comprobar si las retinografías tenían la calidad adecuada y evaluar el estado de la retina del paciente. El oftalmólogo rellenó un formulario tipo base de datos en la aplicación HPDoctor versión 2.17.0.01 (Hewlett-Packard Español SA), que permite tanto generar informes clínicos (que eran enviados al médico solicitante de la exploración vía e-mail) como explotar los datos almacenados para análisis estadísticos ulteriores.

Cuando el oftalmólogo detectó patología oftalmológica que requería exploración especializada, el paciente fue citado directamente en el hospital para realización de las pruebas diagnósticas complementarias correspondientes. Si las retinografías fueron 
no valorables el paciente fue citado para exploración funduscópica y los resultados introducidos en la aplicación informática.

Los datos referentes a la retinopatía diabética se recogieron siguiendo la clasificación realizada por la American Academy of Oftalmology (14) que divide a la RD en: RD no aparente, RD no proliferativa (RDNP) leve, RDNP moderada, RDNP severa y RD proliferativa (RDP). El edema macular (EM) lo subdivide en EM leve, EM moderado y EM severo.

Estos datos fueron introducidos en una tabla de datos Excel. El estudio estadístico fue realizado mediante el programa SPSS versión 11.5 (SPSS inc, Chicago, Illinois, USA).

\section{RESULTADOS}

1.393 pacientes diabéticos fueron citados en consulta para la realización de fotografías de fondo de ojo mediante cámara no midriática, de los cuales asistieron a la consulta 1.189 pacientes y se obtuvieron 1.048 pacientes con formularios telemáticos completos. Fueron incluidos 573 varones y 475 mujeres. La distribución de los pacientes según el tipo de DM que presentaban se puede ver en la figura 1.

De todos los estudios fotográficos realizados, $121(10,09 \%)$ fueron catalogados como no valorables o no quedaron registradas sus imágenes, pero los hallazgos de la exploración de FO fueron introducidos en la base de datos tras la exploración funduscópica tradicional.

En la figura 2 señalamos la distribución de los pacientes dependiendo de la existencia de RD y su gravedad. Los pacientes que presentaron algún grado de retinopatía diabética fueron 220 (21\%). Si se dividen estos pacientes según el tipo de DM (fig. 3), se obtiene que el $26,12 \%$ de los pacientes con DM tipo 1 (DM1) tenían algún grado de retinopatía, así

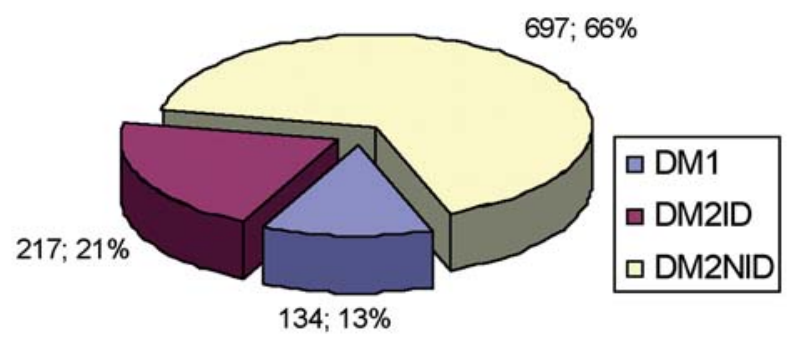

Fig. 1: Distribución de los pacientes según el tipo de DM.

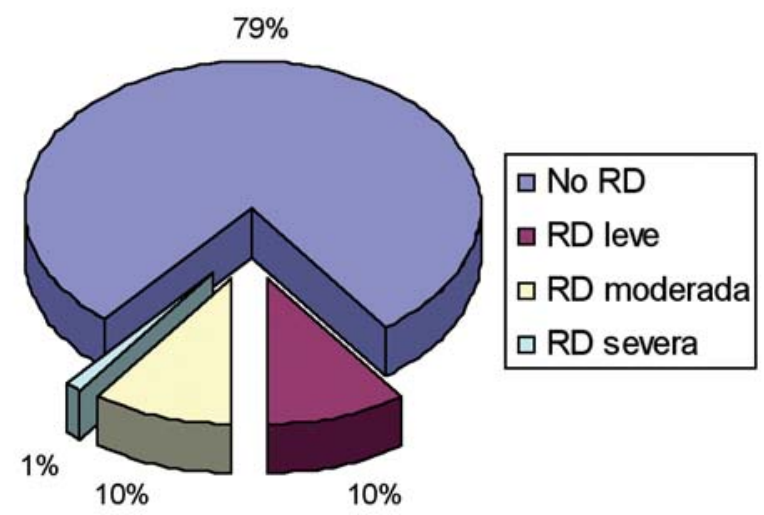

Fig. 2: Prevalencia de RD y gravedad de la misma.

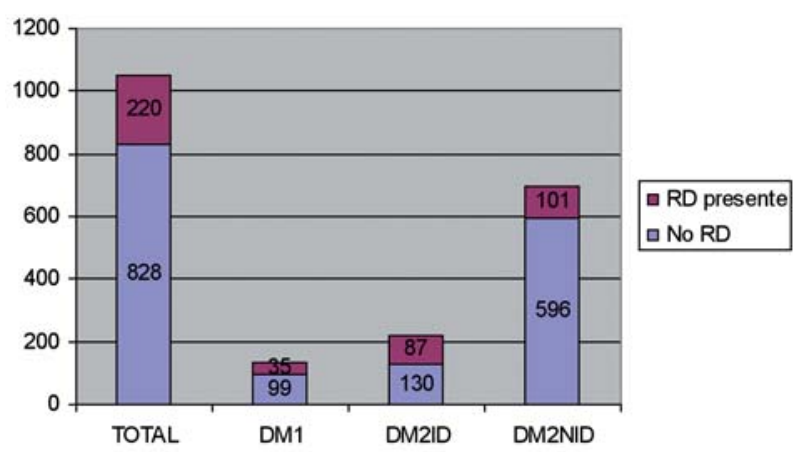

Fig. 3: Número de pacientes afectos de RD según el tipo de $D M$.

como el 40,09\% de los pacientes con DM tipo 2 con tratamiento con insulina (DM2ID) y el 14,49\% de los pacientes con DM tipo 2 sin tratamiento con insulina (DM2NID).

La gravedad de la RD según tipo de DM la podemos ver en la figura 4.

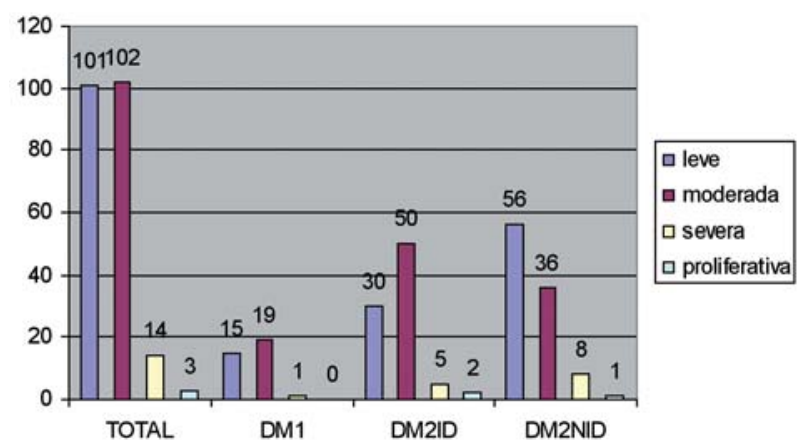

Fig. 4: Número de pacientes con $R D$ y su severidad según el tipo de DM. 


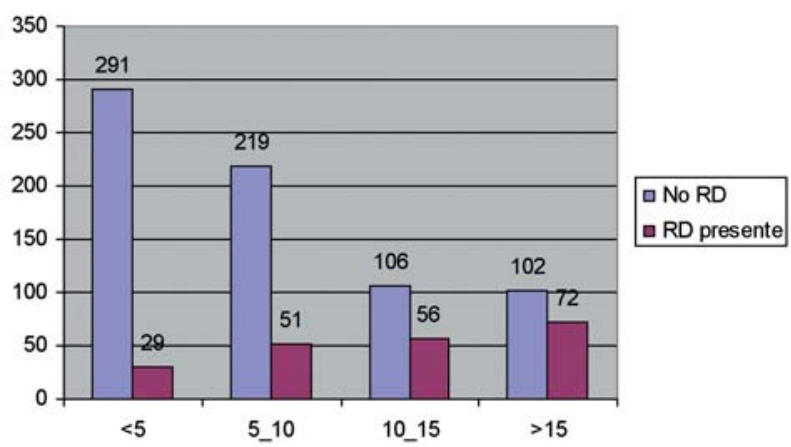

Fig. 5: Número de pacientes con RD en función del tiempo de evolución en años de la DM.

Se realizó un estudio comparativo para observar el comportamiento de la RD respecto al tiempo de evolución de la DM (fig. 5). El 41,38\% de los pacientes diabéticos de más de 15 años de evolución tenían algún grado de RD frente al 15,59\% de los que tenían menos de 10 años de evolución.

Cuando se clasificó la gravedad de RD según el tiempo de evolución de la DM se observaron claras diferencias sobre todo en el grupo de los pacientes con RD moderada (grupo con mayor número de pacientes) como se puede ver en la figura 6 .

Se comparó también la frecuencia de EM dependiendo del tipo de DM. Así existía cualquier grado de EM en el 7,58\% de los DM1, en el 11,74\% de los DM2ID y en el 2,46\% de los DM2NID.

Se realizó un análisis comparativo para observar el comportamiento del EM respecto al tiempo de evolución de la DM (fig. 7).

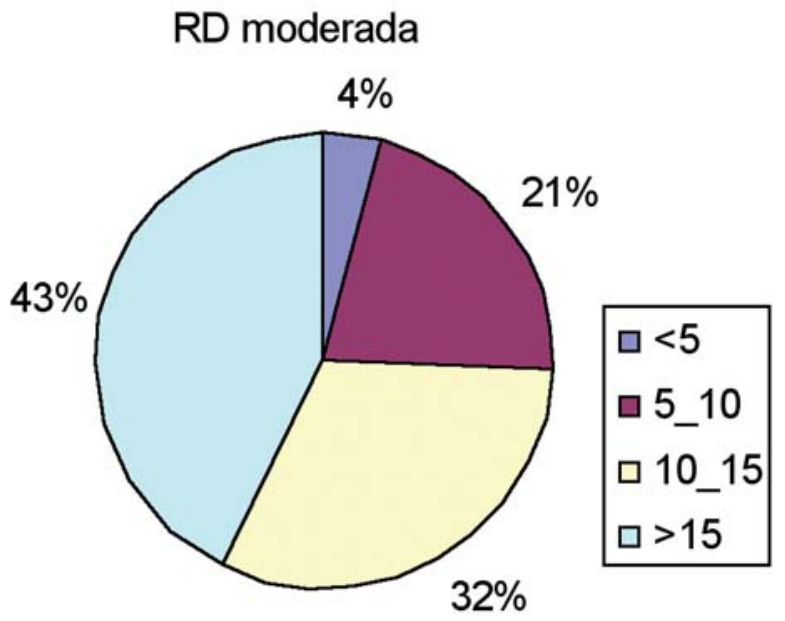

Fig. 6: Tiempo de evolución en años de la DM en pacientes con RD moderada.

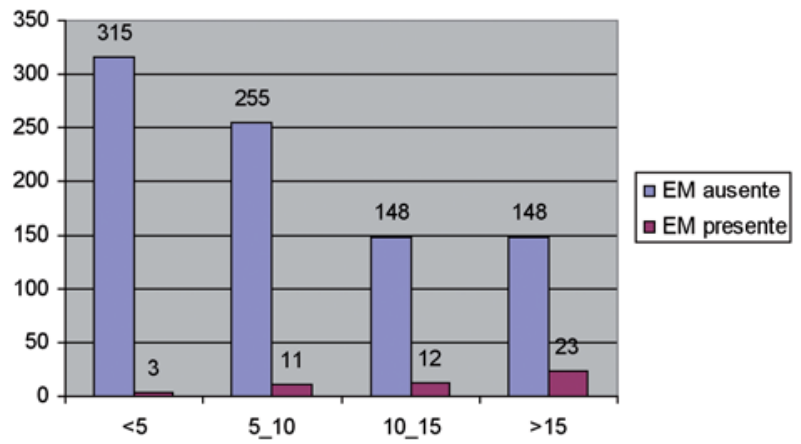

Fig. 7: Número de pacientes con EM en función del tiempo de evolución en años de la DM.

No hubo diferencias estadísticamente significativas cuando se estudió la prevalencia de los tipos de DM por sexo.

\section{DISCUSIÓN}

En este trabajo presentamos los resultados del estudio de prevalencia de 1.393 pacientes diabéticos, siendo uno de los de mayor población estudiada en España con una metodología similar.

La inclusión de los pacientes en este estudio ha sido realizada a partir de pacientes consecutivos remitidos por todos los médicos de atención primaria y endocrinólogos del área 3 de salud. Todos los pacientes diabéticos que pasaron por dichas consultas fueron evaluados. El ideal de este tipo de trabajos es la evaluación de toda la población afecta, evitando sesgos de selección. Nuestro estudio pretende acercarse lo máximo posible a este ideal y con este sistema de selección creemos que se logra.

Otros estudios realizados en España para conocer la prevalencia de $\mathrm{RD}$ han sido realizados sobre muestras que no son representativas de toda la población, como puede ser el estudio de López et al en Valladolid (15) que estudió la población rural o de Santos et al (16) que estudió la población urbana de Badajoz. Otros trabajos sí que han pretendido tener una muestra representativa de la población (17-22) pero en ningún caso participaron todos los centros que llevan el seguimiento de la población diabética, sino que se seleccionaron centros de control diabetológico que fueran, según su criterio, representativos de la población diabética y se calculó el tamaño muestral necesario a partir de de una 
estimación previa de la prevalencia de $\operatorname{RD}(18,20)$. En nuestro caso la participación de todos los médicos de atención primaria y endocrinólogos del área de salud nos permite asegurar que el muestreo es representativo de la población.

La prevalencia de retinopatía diabética en este estudio ha sido del $21 \%$, menor que en el resto de estudios realizados en España exceptuando el de López et al (15). Las diferencias pueden ser debidas a diferencias reales de prevalencia en regiones con distinto control de la DM o a las diferencias entre los sistemas de inclusión de pacientes.

Por otra parte el sistema de clasificación utilizado por nosotros es la Clasificación Clínica Internacional de la Retinopatía Diabética recomendada por la AAO (14) a diferencia de otros estudios que utilizan el sistema de clasificación del ETDRS modificado, por lo que los diferentes criterios podrían explicar las diferentes prevalencias encontradas en las tasas de gravedad de RD y hacer que nuestros datos no fuesen comparables con los de otros estudios.

En cualquier caso esta baja prevalencia denota el buen control de la patología diabética en el Área 3 de la comunidad de Madrid. Este hecho es especialmente significativo en los pacientes con DM1. Otros estudios han encontrado diferencias entre la prevalencia de RD dependiendo de poblaciones y calidad en el control de la DM (16).

La prevalencia de EM en nuestro estudio es de $4,68 \%$, que entra en el rango descrito por otros autores (15-20).

Con este estudio piloto pretendemos conocer la prevalencia de la RD en una muestra de pacientes diabéticos enviados para revisión de fondo de ojo por el médico de atención primaria o el endocrinólogo. Con este método nos aproximamos mucho a lo que sería un estudio poblacional, y buscamos en un futuro cubrir prácticamente toda la población de diabéticos atendidos por el Sistema de Salud de Madrid que vivan en el área sanitaria 3 de la comunidad de Madrid. La muestra poblacional utilizada en este estudio es lo suficientemente grande como para realizar una estimación correcta de la población afecta de RD. El sistema utilizado para ello ha demostrado ser efectivo (5-12). La conjunción de las nuevas tecnologías como las CNM y la telemedicina han permitido, en poco tiempo y optimizando los recursos, la revisión de un gran número de pacientes de una manera cómoda tanto para el paciente como para el oftalmólogo.

\section{BIBLIOGRAFÍA}

1. Fong DS, Aiello L, Gardner TW, King GL, Blankenship G, Cavallerano JD, et al. Diabetic retinopathy. Diabetes Care 2003; 26: S99-S102.

2. Lopez-Bastida J, Cabrera-Lopez F, Serrano-Aguilar P. Sensitivity and specificity of digital retinal imaging for screening diabetic retinopathy. Diabet Med 2007; 24: 403-407.

3. Aiello LP, Gardner TW, King GL, Blankenship G, Cavallerano JD, Ferris FL 3rd, et al. Diabetic retinopathy. Diabetes Care 1998; 21: 143-156.

4. Grading diabetic retinopathy from stereoscopic colour fundus photographs - an extension of the modified Airlie House classification. ETDRS report number 10. Early Treatment Diabetic Retinopathy Study Research Group. Ophthalmology 1991; 98: 786-806.

5. Ahmed J, Ward TP, Bursell SE, Aiello LM, Cavallerano $J D$, Vigersky RA. The sensitivity and specificity of nonmydriatic digital stereoscopic retinal imaging in detecting diabetic retinopathy. Diabetes Care 2006; 29: 2205-2209.

6. Baeza Diaz, M, Gil Guillen V, Orozco Beltran D, Pedrera Carbonell V, Ribera Montes C, Pérez Pons I, et al. Validez. de la cámara no midriática en el cribado de ka retinopatía diabética y análisis de indicadores de riesgo de la retinopatía. Arch Soc Esp Oftalmol 2004;79: 433-441.

7. Scanlon PH, Malhotra R, Greenwood RH, Aldington SJ, Foy $C$, Flatman $M$, et al. Comparison of two reference standards in validating two field mydriatic digital photography as a method of screening for diabetic retinopathy. Br J Ophthalmol 2003; 87: 1258-1263.

8. Massin P, Erginay A, Ben Mehidi A, Vicaut E, Quentel G, Victor Z, et al. Evaluation of a new non-mydriatic digital camera for detection of diabetic retinopathy. Diabet Med 2003;20: 635-641.

9. Chow SP, Aiello LM, Cavallerano JD, Katalinic P, Hock $K$, Tolson A, et al. Comparison of nonmydriatic digital retinal imaging versus dilated ophthalmic examination for nondiabetic eye disease in persons with diabetes. Ophthalmology 2006; 113: 883-840.

10. Lin DY, Blumenkranz MS, Brothers RJ, Grosvenor DM. The sensitivity and specificity of single-field nonmydriatic monochromatic digital fundus photography with remote image interpretation for diabetic retinopathy screening: a comparison with ophthalmoscopy and standardized mydriatic color photography. Am J Ophthalmol 2002;134: 204-213.

11. Heaven CJ, Cansfield J, Shaw KM. The quality of photographs produced by the non-mydriatic fundus camera in a screening programme for diabetic retinopathy: a 1 year prospective study. Eye 1993; 7: 787-790.

12. Bursell SE, Cavallerano JD, Cavallerano AA, Clermont $A C$, Birkmire-Peters D, Aiello LP, et al. Stereo nonmydriatic digital-video color retinal imaging compared to ETDRS 7-standard field 35-mm stereo color photos to diagnose level of diabetic retinopathy. Ophthalmology 2001;108: 572-585.

13. Aldington SJ, Kohner EM, Meuer S, Klein R, Sjolie AK. Methodology for retinal photography and assessment of diabetic retinopathy: the EURODIAB IDDM complications study. Diabetologia 1995; 38: 437-444. 
14. Wilkinson CP, Ferris FL 3rd, Klein RE, Lee PP, Agardh $C D$, Davis $M$ et al. Proposed international clinical diabetic retinopathy and diabetic macular edema disease severity scales. Ophthalmology 2003; 110: 1677-1682.

15. López IM, Díez A, Velilla S, Rueda A, Álvarez A, Pastor $C J$. Prevalence of diabetic retinopathy and eye care in a rural area of Spain. Ophthalmic Epidemiol 2002; 9: 205214.

16. Santos-Bueso E, Fernández-Pérez C, Macarro-Merino A, Fernández-Vigo J. Prevalencia de retinopatía diabética en la ciudad de Badajoz 2002 (Proyecto Extremadura para la prevención de la ceguera). Arch Soc Esp Oftalmol 2007; 82: 153-158.

17. Goldaracena MB, Escudero JM, Arrondo A, Villarubia A, Aramendia B, Iturralde $R$. Prevalencia de retinopatía diabética en una población diabética registrada en atención primaria. Arch Soc Esp Oftalmol 1998; 73: 263-268.

18. Teruel-Maicas C, Fernández-Real JM, Ricart W, ValentFerrer R, Valles-Prats M. Prevalencia de retinopatía dia- bética en la región de Girona. Estudio de factores relacionados. Arch Soc Esp Oftalmol 2005; 80: 85-91.

19. Sender-Palacios MJ, Maceras-Bover M, Vernet-Vernet M, Larrosa Saez P, de la Puente-Martorell ML, Foz-Sala. Aplicación de un método de detección precoz de retinopatía diabética en la Atención Primaria de Salud. Rev Clin Esp 2003; 203: 224-229.

20. Romero P, Del Castillo D. Estudio de prevalencia de retinopatía diabética en la población del Baix Camp (Tarragona). Arch Soc Esp Oftalmol 1996; 71: 261-268.

21. Fernández-Vigo J, Sánchez-Macho J, Díaz-Rey A, Barros $J$, Tome M, Bueno J. The prevalence of diabetic retinopathy in Northwest Spain. An epidemiological study of diabetic retinopathy in Galicia. I. Acta Ophthalmol 1993; 71: 22-26.

22. Scanlon PH, Foy $C$, Malhotra $R$, Aldington SJ. The influence of age, duration of diabetes, cataract, and pupil size on image quality in digital photographic retinal screening. Diabetes Care 2005; 28: 2448-2453. 